\title{
Biochemical Studies on Cerebrospinal Fluid in Patients With Unresponsive Wakefulness Syndrome: Toward a Potential Search for Biomarkers
}

\section{Lijuan Cheng}

International Vegetative State and Consciousness Science Institute, Hangzhou Normal University huang yuehong

Second Affiliated Hospital of Zhejiang University International Medical Center

\section{Yan Luo}

Medical College, Hangzhou Normal University

\section{Hao Yang}

International Vegetative State and Consciousness Science Institute, Hangzhou Normal University

Xiaohua Hu

Wu jing Hospital of Hangzhou

Jianzhong Shen

Shanghai Yongci Rehabilitation Hospital

\section{Wangshan Huang}

International Vegetative State and Consciousness Science Institute, Hangzhou Normal University

\section{Yunzhi Nie}

International Vegetative State and Consciousness Science Institute, Hangzhou Normal University

Haibo Di ( $\nabla$ dihaibo19@aliyun.com)

International Vegetative State and Consciousness Science Institute, Hangzhou Normal University

\section{Research Article}

Keywords: unresponsive wakefulness syndrome, cerebral spinal fluid, biomarkers, proteomic analysis

Posted Date: November 8th, 2021

DOI: https://doi.org/10.21203/rs.3.rs-1012835/v1

License: (1) This work is licensed under a Creative Commons Attribution 4.0 International License. Read Full License 


\section{Abstract}

Background: This study aimed to examine and screen patients for potential biomarkers for the diagnosis of unresponsive wakefulness syndrome (UWS).

Methods: Patients with UWS, patients who regained consciousness (RC; patients in a minimally conscious state), and patients who had emerged from the minimally conscious state were evaluated using the Coma Recovery Scale-Revised (CRS-R). Cerebrospinal fluid (CSF) was collected from five healthy controls and 10 patients ( 5 UWS; $5 \mathrm{RC}$ ). Two-dimensional electrophoresis and proteomic analysis were used to examine and identify differentially expressed proteins in the CSF.

Results: Compared with the control group, there were at least six proteins expressed at higher levels and two proteins expressed at lower levels in the CSF of the RC group than in the CSF of the UWS group. However, expression of vitamin D-binding protein (VDP) showed a further increase of 4.52 -fold, that of DNA replication licensing factor mini-chromosome maintenance 4 (MCM4) showed a reduction of -20.61fold, and that of hemoglobin subunit beta reversed to -8.34-fold in the UWS group. Another converse expression was of protein coenzyme Q-binding protein COQ10 homolog A (COQ10A), which was -4.31fold in the RC group and 1.51-fold in the UWS group, compared to the control group. Expression of other proteins demonstrated an ascending trend in the RC group compared to the control group.

Conclusions: The three differentially expressed proteins, VDP, DNA replication licensing factor MCM4, and hemoglobin subunit beta, may be involved in consciousness maintenance. This study identified potential biomarkers for UWS and will provide new insights into the pathogenesis of UWS.

\section{Introduction}

Patients suffering from severe brain injury may fall into a coma and develop a variety of different clinical awareness states. Some patients progress to unresponsive wakefulness syndrome (UWS) [1], in which the patient appears to be awake, with cycles of eye closure and opening. These patients show no signs of awareness, defined in terms of "purposeful or voluntary responses." Other patients may improve and occasionally present inconsistent but reproducible "purposeful or voluntary responses." These patients are considered to be in a minimally conscious state (MCS), and their limited but definite behaviors are considered evidence of self or environmental awareness [2]. Current studies mostly focus on the diagnosis, prognosis, differentiation between UWS and MCS, management of the MCS, or promotion of recovery from coma [3-7]. Given the ethical limitations of research in these areas, the molecular mechanisms of UWS or MCS are lacking. Thus, to date, few studies have explored the biological mechanism of UWS or MCS, and biomarkers for these conditions remain undetected.

Cerebrospinal fluid (CSF) is composed of a few small molecular substances such as salt, and peptides, as well as relatively large molecules, including proteins and enzymes, among others. Because CSF is located in the cerebral ventricle and subarachnoid space and is in close contact with the extracellular space of the brain, it is believed that many of the biochemical modifications in the brain should be 
reflected in the CSF, and biomarkers originating from it may shed light on the pathophysiology and severity of diseases. Therefore, CSF has been investigated with the aim of understanding the pathogenesis of neurological diseases such as Parkinson's disease and Huntington's disease [8-10]. In general, UWS/MCS patients experience traumatic brain injury (TBI) or cerebral vascular accidents, accompanied by disruption of the blood-brain barrier. The detection of protein biomarkers for TBI will not only reveal the severity of the injury but also predict the type of injury and prognosis in patients with disorders of consciousness (DOC). In addition, TBI biomarkers can also be used to monitor patients' responses to treatment [11]. CSF studies have shown that major components extracted from CSF inflammation profiles can predict the prognosis of severe craniocerebral injury survivors [12]. In addition, age, brain-derived neurotrophic factor (BDNF) genotype, and BDNF levels are also considered mortality/outcome predictors [13]. In addition to CSF studies, protein differences in the plasma have been used to identify new biomarkers for diagnosis and prognosis. Bao et al. screened differentially expressed proteins (DEPs) in patients with chronic consciousness disorder and healthy individuals and found that alpha-1 antitrypsin level was correlated with the level of consciousness measured using the Coma Recovery Scale-Revised (CRS-R) [14] scores and could be predictive of patients' outcomes [15]. However, whether there are other potential biomarkers of UWS in the CSF remains unknown. Accordingly, we examined and screened various biomarkers with the aim of identifying appropriate biomarkers for UWS compared to patients who regained consciousness (RC).

\section{Methods And Materials}

\section{Participants}

Patients were recruited from the Wujing Hospital of Hangzhou City (Hangzhou, China) from February 2012 to June 2013. Ten patients with TBI were included, and five healthy controls without any history of prior neurological diseases were also recruited (Table 1). Exclusion criteria for the healthy controls included (1) documented history of tumor, (2) presence of infections, and (3) any other polytrauma[14]. 
Table 1

Clinical data of control and DOC patients

\begin{tabular}{|c|c|c|c|c|c|c|}
\hline Groups & Patient ID & Sex & Age(years) & Time from injury (days) & CRS-R score & Average \\
\hline \multirow[t]{5}{*}{ Control } & 1 & M & 60 & - & - & - \\
\hline & 2 & $\mathrm{~F}$ & 30 & - & - & - \\
\hline & 3 & $\mathrm{~F}$ & 26 & - & - & - \\
\hline & 4 & $M$ & 45 & - & - & - \\
\hline & 5 & $\mathrm{~F}$ & 52 & - & - & - \\
\hline \multirow[t]{5}{*}{ UWS } & 1 & M & 35 & 733 & $5(1-0-2-1-0-1)$ & \multirow[t]{5}{*}{3.6} \\
\hline & 2 & M & 53 & 118 & $4(0-0-2-1-0-1)$ & \\
\hline & 3 & $\mathrm{~F}$ & 63 & 147 & $5(1-1-1-1-0-1)$ & \\
\hline & 4 & M & 37 & 22 & $3(0-0-2-1-0-0)$ & \\
\hline & 5 & M & 55 & 26 & $1(0-0-0-1-0-0)$ & \\
\hline \multirow[t]{5}{*}{$\mathrm{RC}$} & 1 & $\mathrm{~F}$ & 49 & 56 & $16(3-4-5-2-0-2)$ & \multirow[t]{5}{*}{21.6} \\
\hline & 2 & $\mathrm{~F}$ & 60 & 124 & $23(4-5-6-3-2-3)$ & \\
\hline & 3 & M & 55 & 40 & $23(4-5-6-3-2-3)$ & \\
\hline & 4 & $M$ & 75 & 57 & $23(4-5-6-3-2-3)$ & \\
\hline & 5 & $M$ & 59 & 167 & $23(4-5-6-3-2-3)$ & \\
\hline
\end{tabular}

\section{Procedure}

The behavioral assessment and collection of CSF samples were performed on the day of admission to our rehabilitation unit and within $12 \mathrm{~h}$ after admission. The patients were first assessed by classical clinical neurological examination, and the level of consciousness was diagnosed using the CRS-R. The collection of CSF samples and the CRS-R assessment were performed 3 months after admission on the same day (Figure 1).

The study was approved by the ethics committee of the Rehabilitation Center for Brain Damage of Wujing Hospital. Written informed consent was obtained from the patients' legal surrogates or from health controls.

\section{Behavioral assessments}


The behavior of all DOC patients was assessed using the CRS-R scale, which consists of 23 hierarchically arranged items that comprise six subscales addressing auditory, visual, motor, promotor/verbal, communication, and arousal processes [15].

\section{CSF Sample Collection and Handling}

CSF collection was performed according to the routine protocol at the Wujing Hospital of Hangzhou City. Briefly, $8 \mathrm{ml}$ of CSF was collected in siliconized polypropylene tubes. The first $2 \mathrm{ml}$ of CSF was sent to the local laboratory for routine testing in the hospital. The rest was centrifuged at $2000 \mathrm{~g}$ for $10 \mathrm{~min}$ at room temperature, transferred into new $15-\mathrm{mL}$ precooled siliconized polypropylene tubes, and stored at $-80^{\circ} \mathrm{C}$ for further analysis. Since CSF biomarkers remain stable for at least two freeze-thaw cycles [16], the freeze-thaw in the present study was not expected to contribute to the total variance associated with each biomarker measurement.

\section{Protein extraction}

After thawing, CSF samples of the same volume from the same group were mixed. CSF $(20 \mathrm{ml})$ was added to the centrifuge tube and sonicated intermittently for $10 \mathrm{~s}$, followed by a $10 \mathrm{~s}$ cooling period. This process was repeated 15 times. After sonication, the samples were centrifuged at $12000 \mathrm{~g}$ for 30 min at $4^{\circ} \mathrm{C}$. The supernatant was removed into Millipore Amicon and centrifuged at $4400 \times \mathrm{g}$ for $30 \mathrm{~min}$; the filtered liquid was collected into a new tube, and the sample protein concentration was detected using a 2-D Quant kit (GE Healthcare, UK).

\section{Two-dimensional electrophoresis}

Two-dimensional electrophoresis was performed as previously described [17]. Briefly, $400 \mu \mathrm{g}$ of protein sample from CSF was diluted with a hydration solution (8 mol/L urea, $13 \mathrm{mmol} / \mathrm{L} \mathrm{DTT,} \mathrm{0.5 \%} \mathrm{IPG} \mathrm{buffer,}$ $2 \%$ CHAPS, $0.002 \%$ bromophenol blue) to a total volume of $450 \mu$ and loaded on the IPG Ready Strip (Bio-rad, USA) with the method of in-gel swelling, with two replicates per sample. After isoelectric focusing electrophoresis was performed with the following protocols ( $30 \mathrm{~V}, 12 \mathrm{~h} ; 500 \mathrm{~V}, 500 \mathrm{Vhr} ; 1000 \mathrm{~V}$, $1000 \mathrm{Vhr} ; 8000 \mathrm{~V}, 8000 \mathrm{Vhr}$ ), the strips were equilibrated for $15 \mathrm{~min}$ in equilibrium liquid 1 (6 mol/L urea, $50 \mathrm{mmol} / \mathrm{L}$ Tris, $1 \%$ DTT, $30 \%$ glycerol, $2 \%$ SDS, $0.002 \%$ bromophenol blue) and equilibrium liquid 2 (6 $\mathrm{mol} / \mathrm{L}$ urea, $50 \mathrm{mmol} / \mathrm{L}$ Tris, $4 \%$ iodoacetamide, $30 \%$ glycerol, $2 \%$ SDS, $0.002 \%$ bromophenol blue). The secondary dimensional separation was performed using 12.5\% SDS-PAGE gel electrophoresis (30 W for $45 \mathrm{~min}$ followed by incubation at $90 \mathrm{~W}$ for $6 \mathrm{~h}$ ).

\section{Image scanning and software analysis}

At the end of electrophoresis, the gels were transferred to a staining box for silver staining. The obtained electrophoretograms of different samples were scanned using a UMAX Image Scanner (GE Healthcare, UK) at 300 pixels per square inch, and then analyzed using Image Master 2D Plantinum 6.0, software (GE Healthcare, UK) for point identification, background elimination, point matching, and analysis of differential proteins as described [17]. Each sample was analyzed in triplicate to reduce experimental errors. Scanning and analysis software (Mascot,v2.5) was used to scan and analyze the silver-stained 
gel, with a scanning resolution of $200 \mathrm{dpi}$. Intra-group matching was performed first, and inter-group matching was completed. Proteins with ratios greater than 1.5-fold [18] or less than 1.5-fold among groups were selected as differentially expressed proteins $(p<0.05)$.

\section{In gel digestion and mass spectrometry}

The interesting protein spots were cut off from the 2-DE gels after sliver staining and were washed three times with Milli-Q water followed by the following protocols. First, every spot was destained by washing with a 1:1 solution of $30 \mathrm{mM}$ potassium ferricyanide and $100 \mathrm{mM}$ sodium thiosulfate, and then equilibrated in $200 \mathrm{mM}$ ammonium bicarbonate for $20 \mathrm{~min}$. Next, the spots were dehydrated with acetonitrile after washing twice with Milli-Q water and then dried in a SpeedVac (Eppendorf, USA) for 30 min. Subsequently, the spots were rehydrated with $5 \mu$ trypsin solution $\left(20 \mathrm{ng} / \mu \mathrm{l}\right.$ in $\left.200 \mathrm{mM} \mathrm{NH}_{4} \mathrm{HCO}_{3}\right)$ and incubated at $37{ }^{\circ} \mathrm{C}$ for $12 \mathrm{~h}$. Finally, after washing twice with $30 \mu \mathrm{l}$ of $50 \%$ acetonitrile and $5 \%$ trifluoroacetic acid, $1 \mu \mathrm{l}$ of the peptide mixture was mixed with an equal volume of cyano-4hydroxycinnamic acid ( $10 \mathrm{mg} / \mathrm{ml}$; Sigma, USA) saturated with $50 \%$ acetonitrile in $0.05 \%$ trifluoroacetic acid, and detected by a matrix-assisted laser desorption/ionization time of light matrix-assisted laser desorption/ionization time of flight mass spectrometry (MALDI-TOF- Mass Spectrometer) (4800 Proteomics Analyzer, Applied Biosystems, USA) as described [17].

\section{Results}

\section{Population}

Ten patients with TBI (7 males), aged $-35-60$ years, were included, and the time since injury ranged from 22 to 733 days. Five healthy controls ( 2 males; mean age $52.5 \mathrm{y}$ ) without any history of prior neurological diseases were also recruited (Table 1). When reassessed at 3 months, 5 patients appeared to be in UWS, as demonstrated by sustained unconsciousness with an average CRS-R score of 3.6 (UWS group; 4 men and 1 woman), and the other 5 patients regained signs of consciousness with an average CRS-R score of 21.6 (RC group; 3 males and 2 females)[14]. Detailed information about the patients' clinical data and CRS-R scores are presented in Table 1.

\section{Differential analysis of proteome}

We investigated the proteome profile in the CSF of healthy subjects, patients in the RC group, and in the UWS group using two-dimensional electrophoresis and gel-based proteomics technology. There were at least six protein spots (spots 1, 3, 4, 5, 7, and 8) expressed at higher levels and two protein spots (spots 2 and 6) expressed at lower levels on the gel of CSF from the RC group. These protein spots for the control, $\mathrm{RC}$, and UWS groups are shown in Figure 2A.

\section{Identification of differently expressed proteins}

MALDI-TOF-MS was used to identify the differentially expressed proteins. Eight proteins were detected: vitamin D-binding protein (VDP), coenzyme Q-binding protein C0Q10 homolog A (COQ10A), 
apolipoprotein A-IV (Apo A-IV), transthyretin (TTR), zinc finger BED domain-containing protein 1 (ZBED1), DNA replication licensing factor MCM4 (MCM4), keratin type Il cytoskeletal 1 (Krt1), and hemoglobin subunit beta $(H G \beta)$. Detailed information regarding these proteins is provided in Table 2. The available proteins were compared among the three groups (Table 3 ).

Table 2

Information about the differently expressed proteins in the CSF

\begin{tabular}{|lllll|}
\hline POS & Protein name & Mr & pl & Database Number \\
\hline 1 & Vitamin D-binding protein & 52929 & 5.4 & sp|P02774|VTDB_HUMAN \\
\hline 2 & $\begin{array}{l}\text { Coenzyme Q-binding protein COQ10 } \\
\text { homolog A, mitochondria }\end{array}$ & 27668.1 & 9.81 & sp|Q96MF6|CQ10A_HUMAN \\
\hline 3 & Apolipoprotein A-IV & 45371.5 & 5.28 & sp|P06727|APOA4_HUMAN \\
\hline 4 & Transthyretin & 15877 & 5.52 & sp|P02766|TTHY_HUMAN \\
\hline 5 & $\begin{array}{l}\text { Zinc finger BED domain-containing protein } \\
1\end{array}$ & 78105.6 & 5.79 & sp|096006|ZBED1_HUMAN \\
\hline 6 & DNA replication licensing factor MCM4 & 96497.6 & 6.28 & sp|P33991|MCM4_HUMAN \\
\hline 7 & Keratin, type II cytoskeletal 1 & 65999 & 8.15 & sp|P04264|K2C1_HUMAN \\
\hline 8 & Hemoglobin subunit beta & 15988.3 & 6.75 & sp|P68871|HBB_HUMAN \\
\hline
\end{tabular}

POS: position of series; Mr: Relative molecular mass; pl: isoelectric point; CSF: Cerebrospinal fluid

Table 3. Comparison of the eight proteins among the three inter-groups

\begin{tabular}{lllllllll}
\hline & 1 & 2 & 3 & 4 & 5 & 6 & 7 & 8 \\
\hline $\begin{array}{l}\text { UWS versus } \\
\text { controls }\end{array}$ & 4.52 & 1.51 & 2.12 & 3.51 & 2.37 & -20.61 & $/$ & -8.34 \\
\hline RC versus & 2.25 & -4.31 & 3.29 & 8.24 & 4.24 & -2.54 & 8.9 & 7.38 \\
controls & & & & & & & & \\
\hline UWS versus & -2 & -6.51 & 1.55 & 2.35 & 1.79 & -8.11 & 7.29 & 61.63 \\
RC
\end{tabular}

1:VDP, Vitamin D-binding protein; 2:COQ10A, Coenzyme Q-binding protein COQ10 homolog A, mitochondria; 3:Apo A-IV, Apolipoprotein A-IV; 4:TTR, Transthyretin; 5:ZBED1, Zinc finger BED domain-containing protein 1; 6:MCM4, DNA replication licensing factor MCM4; 7:Krt1, Keratin, type II cytoskeletal 1; $8: \mathrm{HG} \beta$, Hemoglobin subunit beta.

UWS: unresponsive wakefulness syndrome; RC: regained consciousness

As shown in Figure 2B and Figure 3, compared to the healthy control group, the expression of VDP, Apo AIV, TTR, ZBED1, Krt1, and HG $\beta$ increased in the RC group by 2.25-fold, 3.29-fold, 8.24-fold, 4.24-fold, 8.9fold, and 7.39-fold, respectively. In contrast, levels of COQ10A and MCM4 were 4.31-fold lower and 2.54- 
fold lower in the RC group than in the control group, respectively. To explore the potential biomarkers that may indicate the consciousness status or evaluation of therapeutic efficacy in UWS patients, we further analyzed the expression of these differentially expressed proteins in the UWS group. As shown in Figure $2 \mathrm{~B}$ and Figure 3, the expression of VDP showed a further increase as high as 4.52 -fold in the UWS group compared to the healthy group, and the expression of the other up-regulated proteins demonstrated a 1.55-fold decrease in Apo A-IV, 3.51-fold in TTR, 2.37-fold in ZBED1, and 1.22-fold in Krt1. The expression of $\mathrm{HG} \beta$ was reversed to as low as -8.34 -fold in the UWS group. In addition, levels of COQ10A increased 1.51 -fold in the UWS group compared to the control group, although it was 4.31 -fold lower in the RC group than in the control group. Finally, based on the 2.54-fold downregulation in the RC group, the expression of MCM4 showed a further reduction of -20.61-fold in UWS patients (Figures 2B and 3). Taken together, the three differentially expressed proteins, VDP, MCM4, and $H G \beta$, may be involved in not only brain injury, but also consciousness maintenance.

\section{Discussion}

\section{Vitamin D-binding protein}

VDP, also known as Gc-globulin, has a molecular weight of approximately $58 \mathrm{kDa}$, belongs to the albumin gene family, and is primarily synthesized in the liver [19]. It is a secretory protein that has been detected in plasma and CSF at high concentrations [20,21]. VDP is a multifunctional protein because it contains binding sites for several proteins and molecules, including vitamin D, C5a, actin, fatty acids, and endotoxins. Thus, the primary function of VDP is to act as a transport protein for vitamin $D$ and its metabolites or fatty acids [22]. In addition, it exerts several other important physiological functions, such as macrophage modulation, osteoclast activation, and chemotaxis [22]. One of the most important roles of VDP is to scavenge extracellular toxic actin, which is released into the extracellular space after cell death; VDP interacts with and sequesters monomeric actin and inhibits its polymerization [22]. Several studies have demonstrated that the level of VDP in CSF is increased not only in patients with hepatic failure, diabetes, and multiple sclerosis, but also in those with Alzheimer's disease (AD), suggesting that VDP may act as an actin scavenger [21] and play a role in the pathology of $A D[23,24]$. These findings have prompted researchers to explore the functions of extracellular VDP in AD. Recently, Moon et al. reported that VDP interacts directly with both the A $1-42$ monomer and oligomer, resulting in reduced aggregation of $A \beta$, which results in the suppression of $A D$ pathogenesis in vitro [25]. In an $A \beta$-triggered $A D$ mouse model, VDP administration improved $A \beta$-associated impairment of cognitive function by inhibiting synaptic degeneration [25]. In the present study, we found that levels of VDP in CSF in the RC group were higher than those in the healthy control group, with further improvement in the UWS group. It is understandable that the level of VDP in the UWS group was higher than that in the RC group, which may have a smaller lesion. Whether there is any protein or molecule specific to consciousness that could combine with VDP remains unknown.

\section{DNA replication licensing factor MCM4}


Another protein that may be a good biomarker of the UWS is MCM4, which acts as a component of the Mcm 2-7 complex (Mcm complex) and is the putative replicative helicase essential for "once per cell cycle" DNA replication initiation and elongation in eukaryotic cells [26]. In fact, eukaryotic DNA synthesis initiates from multiple replication origins and progresses through bidirectional replication forks to ensure efficient duplication of the genome. Temporal control of initiation from origins and regulation of replication fork functions are important aspects for maintaining genome stability. Multiple kinasesignaling pathways, including Dbf4-dependent Cdc7 kinase (DDK), cyclin-dependent kinase (CDK), and $\mathrm{Mec} 1$, are involved in these processes by targeting the structurally disordered N-terminal serine/threoninerich domain (NSD) of mini-chromosome maintenance subunit 4 (Mcm4), a subunit of the minichromosome maintenance (MCM) replicative helicase complex [27, 28]. Newly published data by Sheu et al. demonstrated that under replication stress, the temporal pattern of origin activation and DNA replication fork progression are altered in cells with mutations within two separate segments of the Mcm4 NSD. Thus, MCM 4 may be a potential intrinsic regulatory domain that integrates multiple signals to coordinate origin activation and replication fork progression under stress conditions [29]. Another study identified a mutation in MCM4 characterizing a new DNA replication disorder. Aside from a phenotype similar to other DNA repair and replication disorders, including increased chromosomal fragility, pre- and postnatal growth retardation, and variable immune deficiency, one study demonstrated that a mutation in MCM4 in a cohort of patients from the Irish Traveler community also led to adrenal insufficiency, and NK cell deficiency [30]. MCM4 is a part of the MCM2-7 complex as a replicative helicase essential for normal DNA replication and genome stability in all eukaryotes. We observed that the protein levels of MCM4 were lower in the CSF of RC patients compared to controls, and further decreased in UWS patients, suggesting that it is possible that our patients may have an increased risk of neoplastic change or degradation alteration in the brain. Currently, however, there is no data showing a link between MCM4 and neurological diseases. In addition, immunoblotting of MCM4 mutation patient lymphocytes showed loss of the full-length $96-\mathrm{kDa}$ MCM4 protein as predicted from RNA analysis, but also showed evidence of a smaller, $85-\mathrm{kDa}$ MCM4 isoform [30]. Whether there is structural alteration of MCM4 in the CSF of our patients remains to be investigated.

\section{Hemoglobin subunit beta}

In addition, we observed a much higher level (7.39-fold increase) of HG $\beta$ in patients in the RC group compared to the control group, which was reversed in UWS patients (8.34-fold decrease compared to control). Hemoglobin is the main oxygen and carbon dioxide carrier in cells of the erythroid lineage and is responsible for oxygen delivery to the respiratory tissues of the body. $H G \beta$ is a component of a larger protein called hemoglobin, which consists of four globin subunits (a2 32 ) [31]. By detecting slight differences between the oxygenation-sensitive bands of alpha and beta subunits, the beta subunits were found to have a higher affinity for oxygen than the alpha subunits [32], indicating the important role of the beta subunit in gas transportation and oxygen supply.

Functional magnetic resonance imaging ( $\mathrm{fMRI}$ ) and positron emission tomography (PET) are widely used to detect residual brain function in UWS, providing useful information for diagnosis and prognosis, and 
an opportunity to study the neural correlates of consciousness [4, 33-35]. Using PET blood flow studies, Laureys et al. demonstrated that simple noxious somatosensory, auditory and visual stimuli typically activated "lower level" primary cortices in patients in UWS, but failed to show robust activation in higher order associative cortices [5, 7, 36, 37]. In our previous study, fMRI was used to study cerebral activation to the subject's own name uttered by a familiar voice and the association of cortical activation with recovery in UWS patients. Two UWS patients who showed "higher level" activation recovered three months after the fMRI study, while none of the five UWS patients, who only showed "primary level" activation, recovered [3]. Using neuroimaging data as a tool to herald recovery of some level of consciousness has been supported by several other studies $[6,38,39]$. Given that the principle of PET or $\mathrm{fMRI}$ is to detect the hemodynamic signals or oxygen content in blood and the function of hemoglobin subunit beta is to carry oxygen, our findings in the present study indicate that the level of hemoglobin subunit beta may be a potential biomarker for the conscious prognosis of patients with disorders of consciousness.

Although we observed increased levels of Apo A-IV, TTR, ZBED1, and Krt1 and decreased levels of COQ10A in the RC group compared to the control group, their levels decreased to a lesser extent in the UWS group. The role these molecules play in brain injury and whether they could serve as useful markers for UWS remain to be elucidated.

\section{Limitations}

In summary, the present study provides insight into the pathogenesis of UWS and identifies potential biomarkers for UWS. However, this study has some limitations. First, since our study enrolled a small number of patients, the consistency of the results require clarification in subsequent experiments. Indeed, due to ethical issues, access to CSF from patients with disorders of consciousness and from healthy individuals was limited. Another limitation is the number of CRS-R; only one was used for the diagnosis, which represents a rate of potential misdiagnosis of $30 \%$. Future investigations should follow the evolution of these biomarkers from onset to better understand patients' neurological recovery. These limitations restrained our ability to determine the relationship between CSF biomarkers and patient prognosis. Finally, these proteins were not further confirmed by western blotting or ELISA (both methods were used to identify the presence of a substance and analyze protein levels in the samples). Hence, further studies with a large sample, including patients with UWS, as well as those in MCS minus and MCS plus, and a linear modulation of these biomarkers to the level of consciousness are necessary to predict consciousness progression.

\section{Abbreviations}

UWS: Unresponsive wakefulness syndrome

RC: Patients who regained consciousness 
CRS-R: Coma Recovery Scale-Revised

CSF: Cerebrospinal fluid

VDP: Vitamin D-binding protein

MCM4: Mini-chromosome maintenance 4

COQ10A: Coenzyme Q-binding protein COQ10 homolog A

MCS: minimally conscious state

TBI: traumatic brain injury

DOC: disorders of consciousness

BDNF: brain-derived neurotrophic factor

DEP: differentially expressed protein

Apo A-IV: apolipoprotein A-IV

TTR: transthyretin

ZBED1: zinc finger BED domain-containing protein 1

Krt1: keratin type II cytoskeletal 1

$H G \beta$ : hemoglobin subunit beta

POS: position of series

Mr: Relative molecular mass

pl: isoelectric point

DDK: Dbf4-dependent Cdc7 kinase

CDK: cyclin-dependent kinase

NSD: N-terminal serine/threonine-rich domain

Mcm4: mini-chromosome maintenance subunit 4

MCM: mini-chromosome maintenance

fMRI: functional magnetic resonance imaging 
PET: positron emission tomography

\section{Declarations}

\section{Ethics approval and consent to participate}

The study was approved by the Ethics Committee of Hangzhou Normal University and Wujing Hospital, which complies with the Code of Ethics of the World Medical Association (Declaration of Helsinki). Informed consent was obtained from the patients' legal surrogates.

\section{Consent for publication}

Not applicable.

\section{Availability of data and materials}

The datasets used and/or analyzed during the current study are available from the corresponding author on reasonable request.

\section{Competing interests}

The authors have no relevant affiliations or financial involvement with any organization or entity with a financial interest in or financial conflict with the subject matter or materials discussed in the manuscript. This includes employment, consultancies, honoraria, stock ownership or options, expert testimony, grants or patents received or pending, or royalties. The peer reviewers of this manuscript have no relevant financial or other relationships to disclose.

\section{Funding}

This study was supported by the National Natural Science Foundation of China (KG08027), the Science and Technology Department of Zhejiang Province (KZ09037), and Hangzhou Normal University (JTAS2011-01-016).

\section{Authors' contributions}

$\mathrm{YL}$ and $\mathrm{XH}$ were responsible for the study design. $\mathrm{LC}, \mathrm{SJ}, \mathrm{WH}$, and $\mathrm{YNwere}$ responsible for data acquisition, analysis, and interpretation. $\mathrm{HY}$ and $\mathrm{YL}$ drafted the manuscript. HD was responsible for the critical revision of the manuscript. All authors read and approved the manuscript for publication.

\section{Acknowledgments}

We wish to thank WuJing Hospital of Hangzhou for providing some elements of data collection.

\section{References}


1. Laureys S, Celesia GG, Cohadon F, Lavrijsen J, Leon-Carrion J, Sannita WG, Sazbon L, Schmutzhard $\mathrm{E}$, von Wild KR, Zeman A et al: Unresponsive wakefulness syndrome: a new name for the vegetative state or apallic syndrome. BMC Med 2010, 8:68.

2. Giacino JT, Ashwal S, Childs N, Cranford R, Jennett B, Katz DI, Kelly JP, Rosenberg JH, Whyte J, Zafonte RD et al: The minimally conscious state: definition and diagnostic criteria. Neurology 2002, 58(3):349-353.

3. Di HB, Yu SM, Weng XC, Laureys S, Yu D, Li JQ, Qin PM, Zhu YH, Zhang SZ, Chen YZ: Cerebral response to patient's own name in the vegetative and minimally conscious states. Neurology 2007, 68(12):895-899.

4. Laureys S, Owen AM, Schiff ND: Brain function in coma, vegetative state, and related disorders. Lancet Neurol 2004, 3(9):537-546.

5. Boly M, Faymonville ME, Peigneux P, Lambermont B, Damas P, Del Fiore G, Degueldre C, Franck G, Luxen A, Lamy $M$ et al: Auditory processing in severely brain injured patients: differences between the minimally conscious state and the persistent vegetative state. Arch Neurol 2004, 61(2):233-238.

6. Owen AM, Menon DK, Johnsrude IS, Bor D, Scott SK, Manly T, Williams EJ, Mummery C, Pickard JD: Detecting residual cognitive function in persistent vegetative state. Neurocase 2002, 8(5):394-403.

7. Laureys S, Faymonville ME, Degueldre C, Fiore GD, Damas P, Lambermont B, Janssens N, Aerts J, Franck G, Luxen A et al: Auditory processing in the vegetative state. Brain 2000, 123 (Pt 8):15891601.

8. Jimenez-Jimenez FJ, Alonso-Navarro H, Garcia-Martin E, Agundez JA: Cerebrospinal fluid biochemical studies in patients with Parkinson's disease: toward a potential search for biomarkers for this disease. Front Cell Neurosci 2014, 8:369.

9. Hall S, Surova Y, Ohrfelt A, Zetterberg H, Lindqvist D, Hansson O: CSF biomarkers and clinical progression of Parkinson disease. Neurology 2014.

10. Huang YC, Wu YR, Tseng MY, Chen YC, Hsieh SY, Chen CM: Increased prothrombin, apolipoprotein AIV, and haptoglobin in the cerebrospinal fluid of patients with Huntington's disease. PloS one 2011, 6(1):e15809.

11. Yokobori S, Hosein K, Burks S, Sharma I, Gajavelli S, Bullock R: Biomarkers for the clinical differential diagnosis in traumatic brain injury-a systematic review. CNS Neurosci Ther 2013, 19(8):556-565.

12. Kumar RG, Rubin JE, Berger RP, Kochanek PM, Wagner AK: Principal components derived from CSF inflammatory profiles predict outcome in survivors after severe traumatic brain injury. Brain Behav Immun 2016, 53:183-193.

13. Failla MD, Conley YP, Wagner AK: Brain-Derived Neurotrophic Factor (BDNF) in Traumatic Brain Injury-Related Mortality: Interrelationships Between Genetics and Acute Systemic and Central Nervous System BDNF Profiles. Neurorehabil Neural Repair 2016, 30(1):83-93.

14. Zhang Y, Wang J, Schnakers C, He M, Luo H, Cheng L, Wang F, Nie Y, Huang W, Hu X et al: Validation of the Chinese version of the Coma Recovery Scale-Revised (CRS-R). Brain Injury. 2019, 33(4):529533. 
15. Bao W, He F, Gao J, Meng F, Zou H, Luo B: Alpha-1-antitrypsin: a novel predictor for long-term recovery of chronic disorder of consciousness. Expert Rev Mol Diagn 2018, 18(3):307-313.

16. Kang JH, Irwin DJ, Chen-Plotkin AS, Siderowf A, Caspell C, Coffey CS, Waligorska T, Taylor P, Pan S, Frasier $\mathrm{M}$ et al: Association of cerebrospinal fluid beta-amyloid 1-42, T-tau, P-tau181, and alphasynuclein levels with clinical features of drug-naive patients with early Parkinson disease. JAMA Neurol 2013, 70(10):1277-1287.

17. Lu D ZY, Zhu J, Teng Y, Yang Z, Chai H, Wo L: Effective Inhibition of Foam Cells Formation by Tanshione IIA in RAW264.7 Macrophages Induced with LDL Isolated from Hypercholesterolemia Patients: A Proteomic Analysis. Current Proteomics 2014, 11:184-192.

18. Gahoi N., Malhotra D., Moiyadi A., Varma S. G., Gandhi M. N., Srivastava S. (2017). Multi-pronged proteomic analysis to study the glioma pathobiology using cerebrospinal fluid samples. Proteomics Clin. Appl. 1700056.

19. White $\mathrm{P}$, Cooke N: The multifunctional properties and characteristics of vitamin D-binding protein. Trends Endocrinol Metab 2000, 11(8):320-327.

20. Speeckaert M, Huang G, Delanghe JR, Taes YE: Biological and clinical aspects of the vitamin D binding protein (Gc-globulin) and its polymorphism. Clin Chim Acta 2006, 372(1-2):33-42.

21. Gressner OA, Schifflers MC, Kim P, Heuts L, Lahme B, Gressner AM: Questioning the role of actinfree Gc-Globulin as actin scavenger in neurodegenerative central nervous system disease: relationship to S-100B levels and blood-brain barrier function. Clin Chim Acta 2009, 400(1-2):86-90.

22. Meier U, Gressner O, Lammert F, Gressner AM: Gc-globulin: roles in response to injury. Clin Chem 2006, 52(7):1247-1253.

23. Abdi F, Quinn JF, Jankovic J, Mclntosh M, Leverenz JB, Peskind E, Nixon R, Nutt J, Chung K, Zabetian $\mathrm{C}$ et al: Detection of biomarkers with a multiplex quantitative proteomic platform in cerebrospinal fluid of patients with neurodegenerative disorders. J Alzheimers Dis 2006, 9(3):293-348.

24. Zhang J, Sokal I, Peskind ER, Quinn JF, Jankovic J, Kenney C, Chung KA, Millard SP, Nutt JG, Montine TJ: CSF multianalyte profile distinguishes Alzheimer and Parkinson diseases. Am J Clin Pathol 2008, 129(4):526-529.

25. Moon M, Song H, Hong HJ, Nam DW, Cha MY, Oh MS, Yu J, Ryu H, Mook-Jung I: Vitamin D-binding protein interacts with Abeta and suppresses Abeta-mediated pathology. Cell Death Differ 2013, 20(4):630-638.

26. Tye BK: MCM proteins in DNA replication. Annu Rev Biochem 1999, 68:649-686.

27. Sheu YJ, Stillman B: The Dbf4-Cdc7 kinase promotes S phase by alleviating an inhibitory activity in Mcm4. Nature 2010, 463(7277):113-117.

28. Forsburg SL: Eukaryotic MCM proteins: beyond replication initiation. Microbiol Mol Biol Rev 2004, 68(1):109-131.

29. Sheu YJ, Kinney JB, Lengronne A, Pasero P, Stillman B: Domain within the helicase subunit Mcm4 integrates multiple kinase signals to control DNA replication initiation and fork progression. Proc Nat/ Acad Sci U S A 2014, 111(18):E1899-1908. 
30. Hughes CR, Guasti L, Meimaridou E, Chuang CH, Schimenti JC, King PJ, Costigan C, Clark AJ, Metherell LA: MCM4 mutation causes adrenal failure, short stature, and natural killer cell deficiency in humans. J Clin Invest 2012, 122(3):814-820.

31. Belcher JD, Beckman JD, Balla G, Balla J, Vercellotti G: Heme degradation and vascular injury. Antioxid Redox Signal 2010, 12(2):233-248.

32. Nasuda-Kouyama A, Tachibana H, Wada A: Preference of oxygenation between alpha and beta subunits of haemoglobin. Results of multidimensional spectroscopic observation. J Mol Bio/ 1983, 164(3):451-476.

33. Coleman MR, Davis MH, Rodd JM, Robson T, Ali A, Owen AM, Pickard JD: Towards the routine use of brain imaging to aid the clinical diagnosis of disorders of consciousness. Brain 2009, 132(Pt 9):2541-2552.

34. Stender J, Gosseries O, Bruno MA, Charland-Verville V, Vanhaudenhuyse A, Demertzi A, Chatelle C, Thonnard $M$, Thibaut $A$, Heine $L$ et al: Diagnostic precision of PET imaging and functional MRI in disorders of consciousness: a clinical validation study. Lancet 2014, 384(9942):514-522.

35. Di Perri C, Bahri MA, Amico E, Thibaut A, Heine L, Antonopoulos G, Charland-Verville V, Wannez S, Gomez F, Hustinx R et al: Neural correlates of consciousness in patients who have emerged from a minimally conscious state: a cross-sectional multimodal imaging study. The Lancet Neurology 2016, 15(8):830-842.

36. Laureys S, Faymonville ME, Peigneux P, Damas P, Lambermont B, Del Fiore G, Degueldre C, Aerts J, Luxen A, Franck $G$ et al: Cortical Processing of Noxious Somatosensory Stimuli in the Persistent Vegetative State. Neurolmage 2002, 17(2):732-741.

37. Giacino JT, Hirsch J, Schiff N, Laureys S: Functional neuroimaging applications for assessment and rehabilitation planning in patients with disorders of consciousness. Arch Phys Med Rehabil 2006, 87(12 Suppl 2):S67-76.

38. Moritz CH, Rowley HA, Haughton VM, Swartz KR, Jones J, Badie B: Functional MR imaging assessment of a non-responsive brain injured patient. Magn Reson Imaging 2001, 19(8):1129-1132.

39. Wang F, Di H, Hu X, Jing S, Thibaut A, Di Perri C, Huang W, Nie Y, Schnakers C, Laureys S: Cerebral response to subject's own name showed high prognostic value in traumatic vegetative state. $B M C$ Med 2015, 13:83.

\section{Figures}




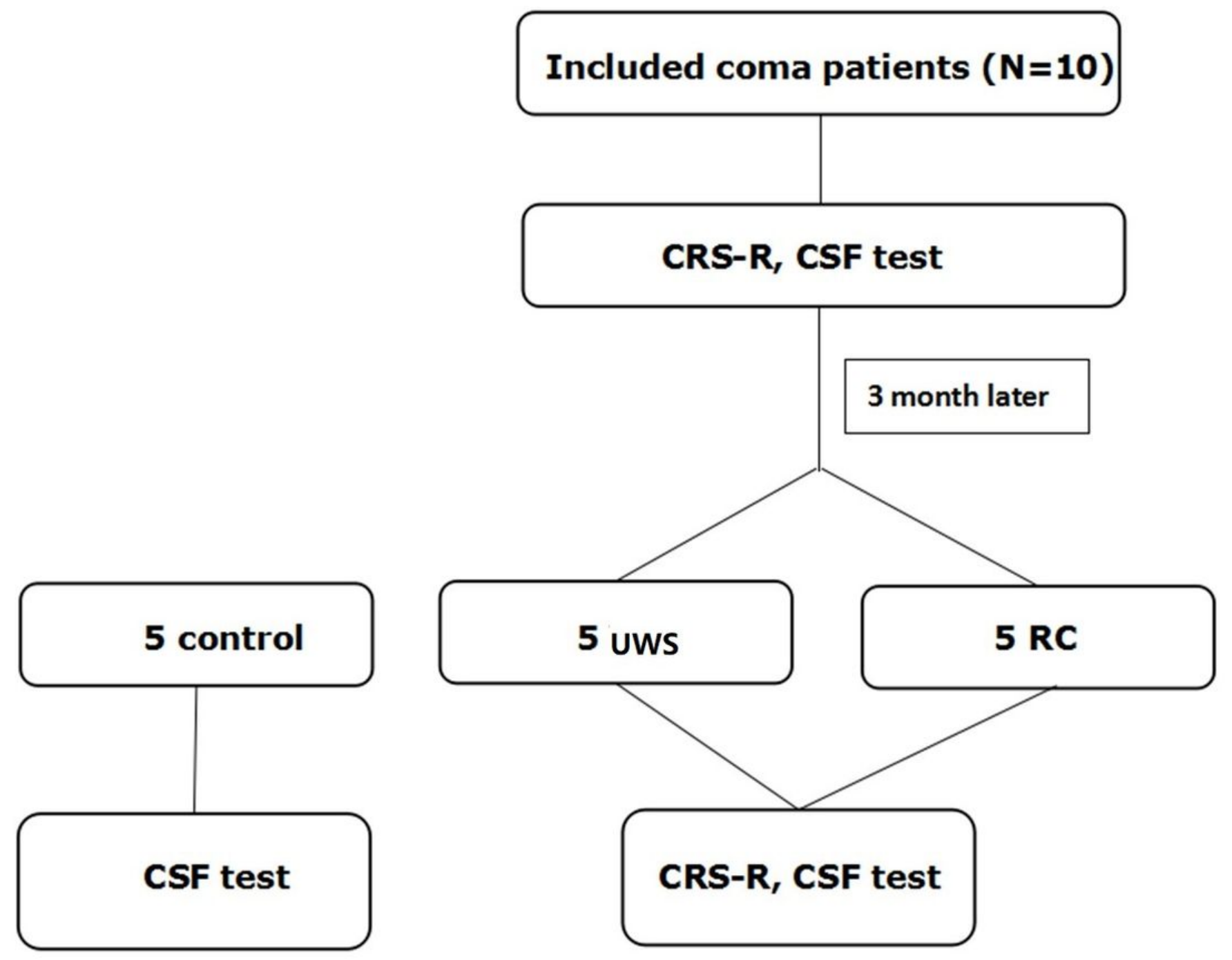

Figure 1

Study procedure. CSF: Cerebrospinal fluid; CRS-R: Coma Recovery Scale-Revised; UWS, unresponsive wakefulness syndrome; RC, regained consciousness 

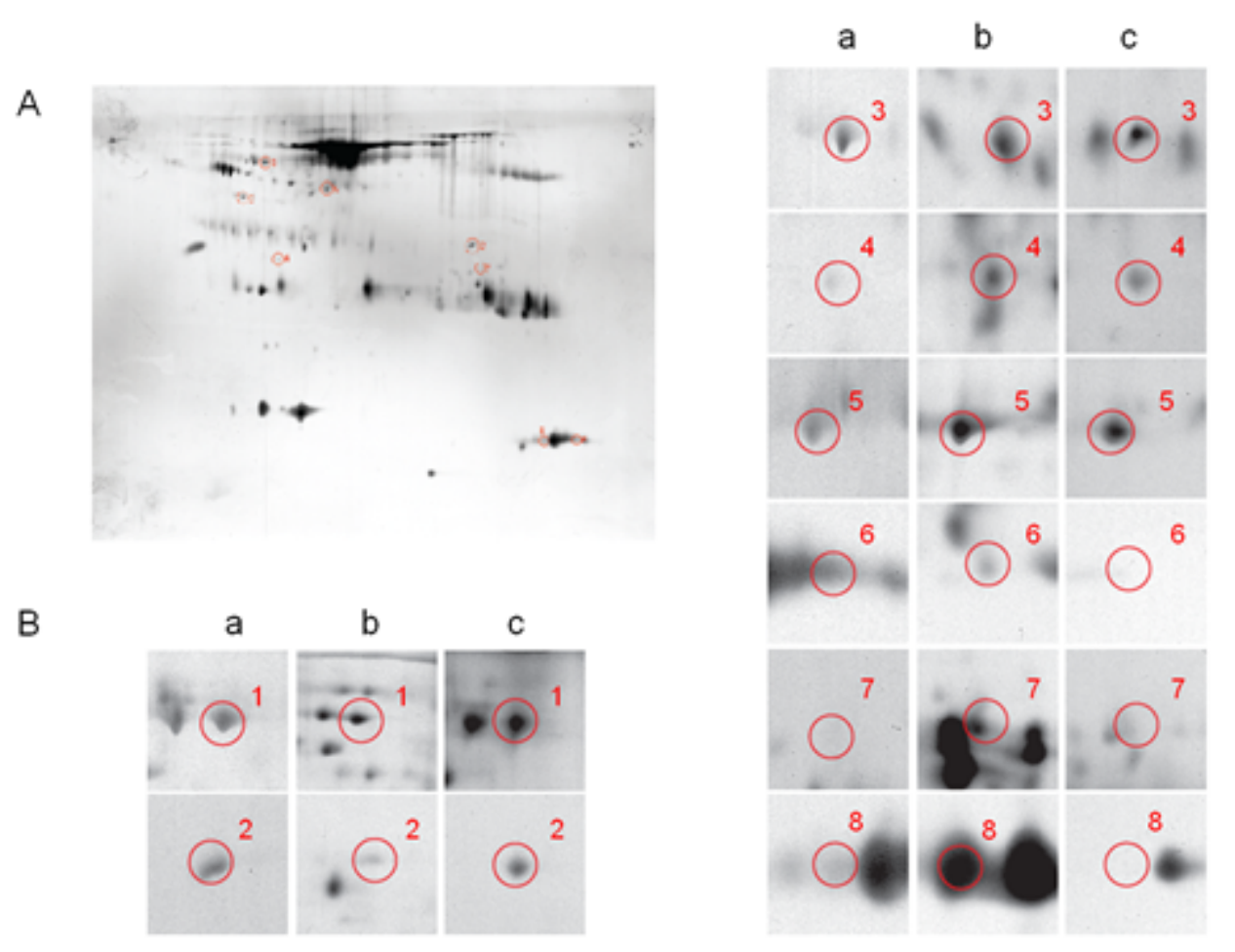

\section{Figure 2}

Representative electrophoresis image of proteins from cerebrospinal fluid of control and patients. Representative two-dimensional electrophoresis image of proteins from cerebrospinal fluid of control and patients with consciousness regaining after treatment and patients in unresponsive wakefulness syndrome after treatment. A, The proteome maps of cerebrospinal fluid. The numbered spots denote proteins that exhibited modified expression levels relative to control group. B, Magnified representative spots showing intensity quantification for differentially expressed proteins; $a$, the control group (healthy subjects); b, patients with consciousness regaining after treatment; $c$, patients in unresponsive wakefulness syndrome after treatment. Number 1 denotes VDP, Vitamin D-binding protein; Number 2 denotes COQ10A, Coenzyme Q-binding protein COQ10 homolog A, mitochondria; Number 3 denotes Apo A-IV, Apolipoprotein A-IV; Number 4 denotes TTR, Transthyretin; Number 5 denotes ZBED1, Zinc finger BED domain-containing protein 1; Number 6 denotes MCM4, DNA replication licensing factor MCM4; Number 7 denotes Krt1, Keratin, type II cytoskeletal 1; Number 8 denotes HG $\beta$, Hemoglobin subunit beta. 
Figure 3

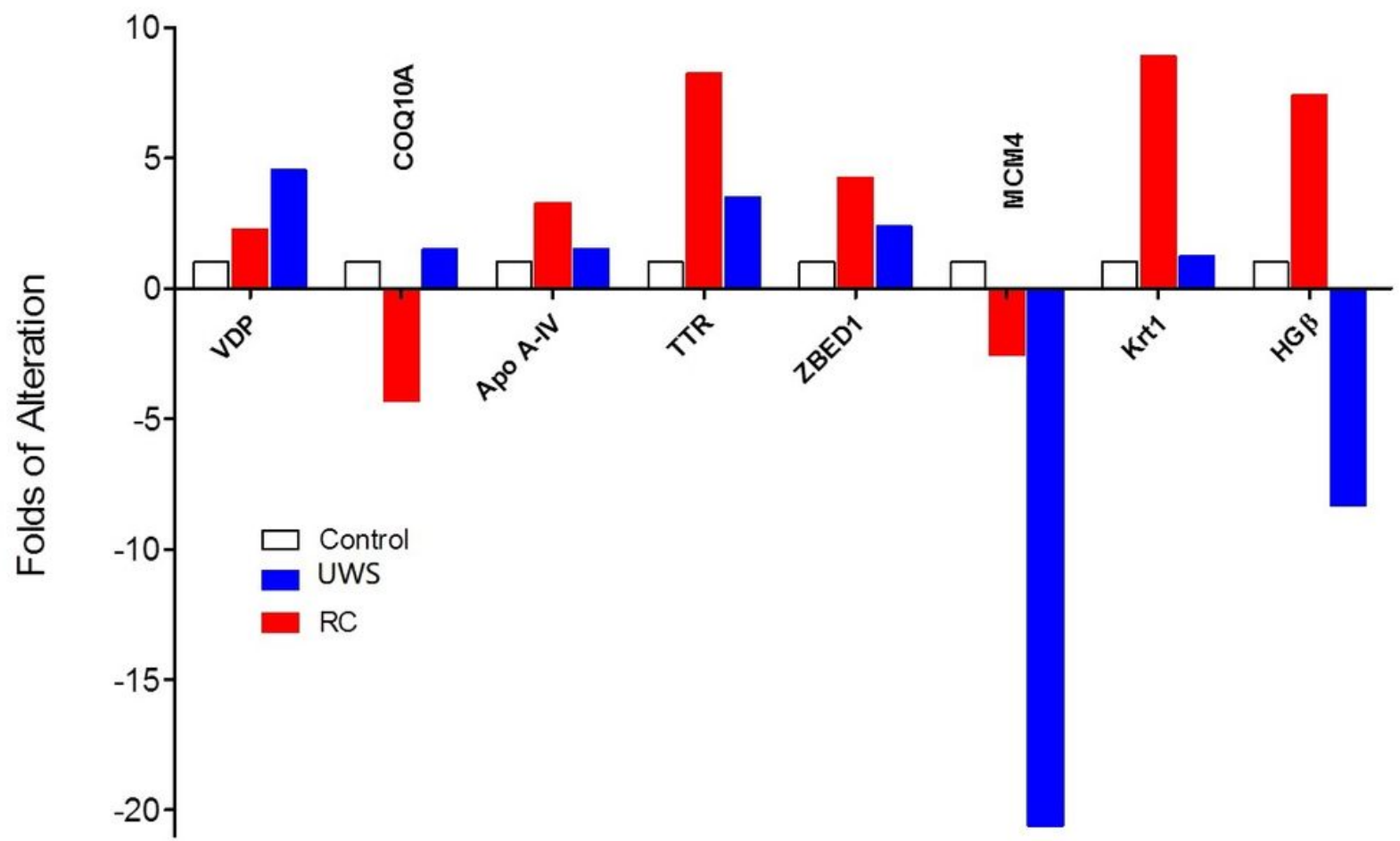

Figure 3

Protein quantification. The analyzed quantification of stain intensity of proteins shown in Figure 2. VDP, Vitamin D-binding protein; COQ10A, Coenzyme Q-binding protein COQ10 homolog A, mitochondria; Apo AIV, Apolipoprotein A-IV; TTR, Transthyretin; ZBED1, Zinc finger BED domain-containing protein 1; MCM4, DNA replication licensing factor MCM4; Krt1, Keratin, type Il cytoskeletal 1; HGß, Hemoglobin subunit beta. 